\title{
Evaluating health-related quality of life in gastric cancer patients in Suzhou, China
}

\author{
Feng-Feng Zhang ${ }^{1 \#}$, Lu Ye ${ }^{2 \#}$, Ni Yin ${ }^{1 \#}$, Feng Xiong ${ }^{1}$, Chun-Hua Zhao ${ }^{3}$, Yan-Bo Zhu ${ }^{1}$ \\ ${ }^{1}$ Department of Oncology, The First Affiliated Hospital of Soochow University, Suzhou, China; ${ }^{2}$ Department of General Medicine, The First \\ Affiliated Hospital of Soochow University, Suzhou, China; ${ }^{3}$ Technology Department, The Affiliated Suzhou Hospital of Nanjing Medical University, \\ Suzhou Municipal Hospital, Gusu School, Nanjing Medical University, Suzhou, China \\ Contributions: (I) Conception and design: FF Zhang, YB Zhu; (II) Administrative support: YB Zhu; (III) Provision of study materials or patients: \\ YB Zhu, F Xiong; (IV) Collection and assembly of data: FF Zhang, L Ye, N Yin; (V) Data analysis and interpretation: FF Zhang, CH Zhao; (VI) \\ Manuscript writing: All authors; (VII) Final approval of manuscript: All authors. \\ "These authors contributed equally to this work. \\ Correspondence to: Yan-Bo Zhu; Feng Xiong. Department of Oncology, The First Affiliated Hospital of Soochow University, Suzhou, China. Email: \\ zhuyanbo@suda.edu.cn; greenlemontea@126.com; Chun-Hua Zhao. Technology Department, The Affiliated Suzhou Hospital of Nanjing Medical \\ University, Suzhou Municipal Hospital, Gusu School, Nanjing Medical University, Suzhou, China. Email: chzhaosuda@163.com.
}

Background Health-related quality of life (HRQOL) has become an important part of the evaluation of clinical efficacy and prognosis in gastric cancer. This study aimed to assess the HRQOL of patients with gastric cancer using the a five-level EuroQol five-dimensional questionnaire (EQ-5D-5L) and explore the factors influencing patients' perceived quality of life. For those significant factors, we can take appropriate measures to intervene to extend patient survival and improve the quality of life.

Methods: A cross-sectional questionnaire survey was administered to 243 patients with gastric cancer in the First Affiliated Hospital of Suzhou University from December 2018 to December 2020. HRQOL was measured by the Chinese version of the EQ-5D-5L. Nonparametric test analyses and a Tobit regression model were used to identify the independent variables associated with the EQ-5D-5L utility scores.

Results: In this research, the mean score was 0.810 , and the median was 0.893 . Approximately $25 \%$ of patients reported no problems at all in any of the five dimensions. Problems in pain and discomfort were the most frequently reported (64.2\%). Nonparametric test analyses showed that patients who did not have health insurance, or who had a history of alcohol use, a family history of cancer, had received surgery only, or had an interval of less than 1 week between taking this survey and their last treatment, demonstrated lower EQ5D-5L scores. The Tobit regression model confirmed that health insurance, family history, and treatment were significantly associated with EQ-5D-5L scores.

Conclusions: The HRQOL of gastric cancer patients can be measured by EQ-5D-5L, and the results may provide a guide for choosing an appropriate individualized treatment plan.

Koywords! Health-related quality of life (HRQOL); five-level EuroQol five-dimensional questionnaire (EQ-5D$5 \mathrm{~L})$; gastric cancer; influencing factors

Submitted May 20, 2021. Accepted for publication Jul 14, 2021.

doi: 10.21037/apm-21-1599

View this article at: https://dx.doi.org/10.21037/apm-21-1599 


\section{Introduction}

Gastric cancer is one of the most common cancers in the world and is a serious threat to human health. In 2018, statistics from the International Agency for Research on Cancer indicated that there were approximately one million new cases and 784,000 deaths from gastric cancer worldwide. Moreover, gastric cancer ranked fifth in incidence and third in mortality rates of all malignant tumors (1). Although the global incidence and mortality of gastric cancer appear to be decreasing, the rates in China are still at a high level. According to statistics released by the National Cancer Registry Center in 2015, the incidence and mortality of gastric cancer in China ranks second among malignant tumors (2). According to the incidence and death of malignant tumors in Suzhou citizens in 2018 officially released by the Suzhou Municipal Health Commission, there were 23,122 new cases of malignant tumors in Suzhou, among which gastric cancer ranked the third, accounting for $11.72 \%$ (only second to lung cancer, accounting for $18.25 \%$, and colorectal cancer, accounting for $11.98 \%$ ). A total of 14,461 malignant tumor deaths occurred, of which gastric cancer accounted for $15 \%$ of all malignant tumors, ranking second only to lung cancer (26\%). In recent 10 years (2009 to 2018), the mortality rate of malignant tumors in Suzhou has been rising steadily (3).

With the continuing advances in medical diagnosis and treatment technologies, overall survival from gastric cancer has significantly improved, but the various psychological and social problems that arise as a consequence of treatment can affect patients' quality of life. Health-related quality of life (HRQOL) has become an important component of the evaluation of clinical efficacy and prognosis in the treatment of gastric cancer.

HRQOL is a subjective, multidimensional concept that includes physical, functional, and mental/emotional health factors related to an individual's health (4). Physical wellbeing refers to the patient's physical state and any treatment side effects; functional health refers to whether a patient's ability to perform daily activities and take care of themselves is affected; emotional aspect refers to whether the patient's mental state and mood are affected. Through the influence on these three aspects, the quality of life of patients will be more or less decreased.

Recently, the use of HRQOL as a clinical outcome measure for cancer treatment has been growing rapidly, reflecting differences in the health status of gastric cancer patients with different clinical conditions and socio-economic characteristics at different stages of the disease (5). Many current studies have shown that the quality of life of patients with gastric cancer is impaired and significantly lower than that of non-cancer people (6-8). There is a need for better monitoring of patients' quality of life to support clinical and public health decision-making. In this way, we can look for factors that affect quality of life and help improve quality of life for cancer patients.

Several instruments measure HRQOL, such as the EuroQol five-dimension (EQ-5D) (9), the short-form six-dimension survey (SF-6D) (10), the health utilities index (HUI) (11) and the European Organization for the Research and Treatment of Cancer quality of life questionnaire (EORTC-QLQ-C30) (12). The EORTCQLQ-C30 was designed for cancer patients in general and the EORTC-QLQ-STO22, developed from it, was the novelist measurement to assess the HRQOL of patients with gastric cancer specifically (13). Of these instruments, the EQ-5D is the simplest, the most widely used and has shown both satisfactory reliability and validity in general patient populations in China (14-17). The EQ-5D, a multi-dimensional evaluation scale, now has two versions: three-level EQ-5D questionnaire (EQ-5D-3L) and fivelevel EQ-5D questionnaire (EQ-5D-5L). The 5L is a new version for describing and evaluating HRQOL. Compared to its predecessor EQ-5D-3L, 5L expands the number of combined health states and shows a lower ceiling effect and a higher sensitivity (18-20). Extensive research has been conducted using the EQ-5D-5L questionnaire to assess HRQOL in different types of patients, including head-andneck cancer (21), breast cancer (22), lung cancer (23), and colorectal cancer (24), but only a few studies have focused on gastric cancer patients in China (25-27). This study used the EQ-5D-5L to investigate the HRQOL of gastric cancer patients and evaluated the influencing factors on patients' perceived quality of life.

We present the following article in accordance with the SURGE reporting checklist (available at https://dx.doi. org/10.21037/apm-21-1599).

\section{Methods}

\section{Study participants}

A cross-sectional questionnaire survey was administered to patients in the First Affiliated Hospital of Soochow University from December 2019 to December 2020. Eligibility criteria were as follows: (I) over 18 years old, (II) 
a pathological diagnosis of gastric cancer, (III) cognitive integrity, and (IV) adequate reading and communication skills. Patients were excluded if they could not understand the survey questions or if they failed or refused to give written consent. Subsequently, 243 patients (170 males and 73 females) were interviewed face-to-face by trained interviewers. This study has been approved by the Ethics Committee of the First Affiliated Hospital of Soochow University (No. 98, 2021) and followed the tenets of the Declaration of Helsinki (as revised in 2013). Informed consent was taken from all the patients.

\section{Measurement of HRQOL}

In this study, HRQOL was estimated by the EQ-5D5L, which has an established Chinese version. The EQ$5 \mathrm{D}$ instrument has demonstrated satisfactory validity and reliability in both general and patient populations in mainland China. It consists of five dimensions: mobility, self-care, usual activities, pain/discomfort, and anxiety/ depression. Each dimension has five levels: 1, no problems; 2, slight problems; 3, moderate problems; 4, severe problems; and 5, extreme problems. The patients were asked to select their perceived severity level from the 1 to 5 scale for different statements. As a result, a total of $3,125\left(5^{5}\right)$ statements can be combined in this system. Each statement can then be converted to a health utility score using the Chinese EQ-5D-5L value set (28). The higher the utility score, the better the quality of life. When all five dimensions are at level 1 , the patient is deemed to be in a state of full health (11111), and the corresponding utility score is 1 , which represents the upper limit of the utility value. The lowest is -0.391 , which represents the worst health status (55555).

\section{Clinical and socioeconomic characteristics}

Data on the clinical features of patients were collected through the medical records, including the stage of gastric cancer [I, II, III, IV; TNM pathological staging according to the American Joint Committee on Cancer (AJCC) 7th edition], treatment (surgery, chemotherapy, or combined surgery and chemotherapy), and complications (e.g., hypertension or diabetes). The socioeconomic characteristics of the respondents were collected from the questionnaire survey, which included gender, age, education level, marital status, and medical insurance. Other data collected concerned lifestyle habits, such as a history of smoking or drinking.

\section{Statistical analysis}

The characteristics and HRQOL of gastric patients assessed by the EQ-5D-5L are summarized using descriptive statistics. The mean and standard deviations (SDs) are used for continuous variables, whereas frequencies and percentages are used for categorical variables. As the EQ5D-5L utility scores of respondents followed a non-normal distribution, nonparametric test analyses were performed to explore the differences in the utility scores of patients with different characteristics, including the Wilcoxon ranksum test for comparison of two independent samples and a Kruskal-Wallis analysis for multiple independent samples. We subsequently established a Tobit regression model between the utility scores and the independent variables that were statistically significant $(\mathrm{P}<0.05)$ in the nonparametric test analyses.

There is a common ceiling effect in EQ-5D instruments when a large number of patients report the highest health state, leading to some utility scores being censored at 1.0 (29-31). When the utility score is used as the dependent variable for statistical modeling, some traditional statistical methods that require the dependent variable to follow a normal distribution are no longer applicable. Therefore, when it comes to censored data, the use of the Tobit regression model is advised (32-34). EpiData3.0 (http:// www.epidata.dk/links.htm) was used to establish the database, and SAS (version 9.4, 2014, SAS Inc., Cary, NC, USA) was used for the statistical analysis.

\section{Results}

\section{Problems reported in the five dimensions of $E Q-5 D-5 L$}

Of the 243 patients in this research, $24.7 \%$ reported no problems in any of the five dimensions. Problems with pain and discomfort were the most frequently reported (64.2\%), which included slight, moderate, severe, and extreme ratings. Problems with anxiety and depression accounted for $35.4 \%$, and self-care problems ranked the lowest (23.9\%), as shown in Table 1.

\section{Overall description of patient scores}

On the whole, the utility scores of gastric cancer patients in this study were encouraging. Almost $25 \%$ of patients 
Table 1 Problems reported by respondents in the five dimensions of the EQ-5D-5L

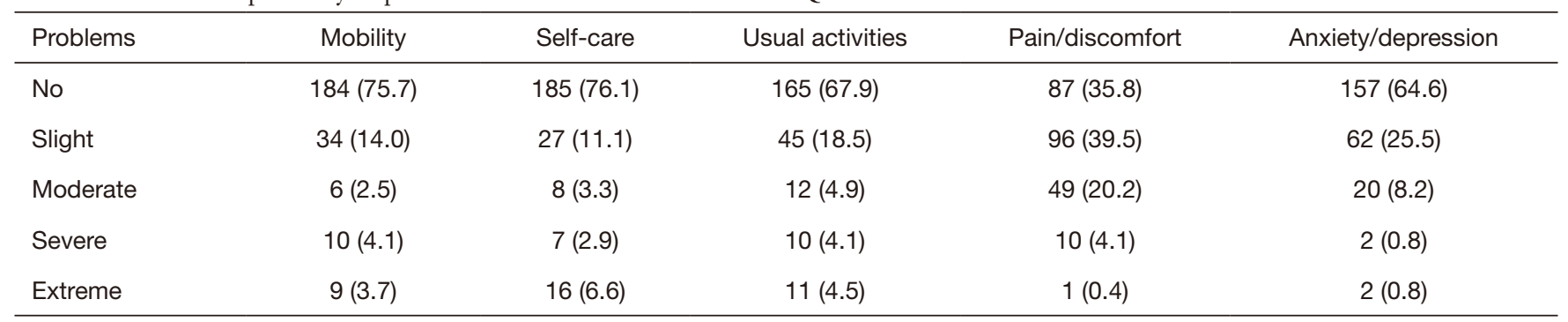

EQ-5D-5L, five-level EuroQol five-dimensional questionnaire.

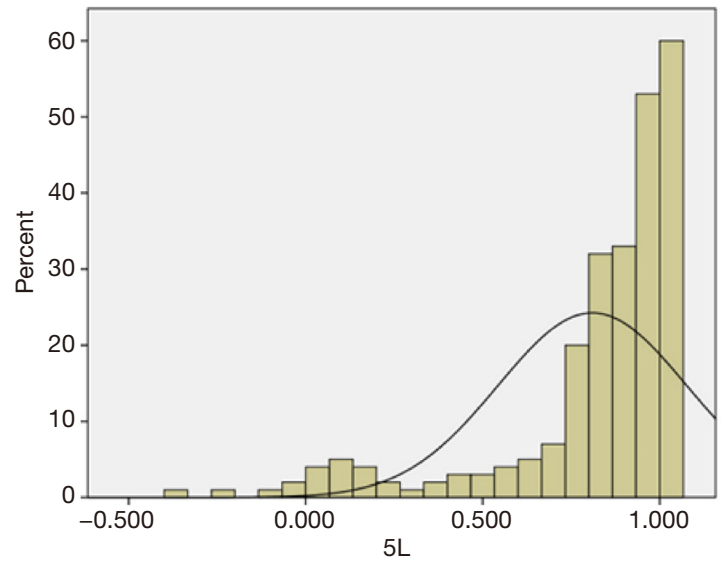

Figure 1 Distribution of the EQ-5D-5L utility scores of patients with gastric cancer. EQ-5D-5L, five-level EuroQol fivedimensional questionnaire.

reported no problem in any EQ-5D domain, and their utility score was 1.0 . The mean score was 0.810 , and the median was 0.893 . The distribution of the utility scores tended towards the right (higher values), as shown in Figure 1.

\section{Comparison of the utility scores in respondents with different characteristics}

Table 2 presents the socioeconomic characteristics of the participants in this study. The mean age of the patients was 62.1 years, and $61.7 \%$ were over 60 years old; males accounted for $70 \%$; the majority lived with their spouses (95.1\%), and $41.6 \%$ were covered by urban residents' health insurance. More than half of the patients reported that they had not smoked (57.2\%) or drunk $68.3 \%$ ) in recent months. Among the 243 patients, only a small percentage (26.3\%) had a family history of cancer.
Among the clinical characteristics, we found that most gastric patients were at stage III (41.2\%) and stage IV (23.5\%). All patients had received medical treatment: $43.2 \%$ had received surgery, $14 \%$ had received chemotherapy alone, and $42.8 \%$ had received combined surgery and chemotherapy. There were 82 patients (33.7\%) who reported that the time between this survey and their last treatment was less than 1 week, while 74 patients (30.5\%) reported an interval of more than 1 month, as shown in Table 3. Many patients reported having no complications: $57.6 \%$ had neither diabetes nor hypertension.

\section{Results of univariate analysis}

As shown in Tables 2,3, those patients who did not have health insurance, had a history of alcohol use, a family history of cancer, received surgery only, or had an interval of less than 1 week between this survey and their last treatment reported lower utility scores $(\mathrm{P}<0.05)$. Meanwhile, no significant differences in the utility scores were found in gender, age, marital status, education levels, complications, or tumor stage $(\mathrm{P}>0.10)$.

\section{Multivariate analysis results}

The Tobit regression model confirmed that health insurance, family history, and treatment were significantly associated with the EQ-5D-5L utility scores $(\mathrm{P}<0.05)$. However, after controlling for other factors, alcohol use and the interval between this survey and the last treatment became insignificant (Table 4).

\section{Discussion}

With the change in medical models, the clinical treatment of gastric cancer no longer simply pursues the extension of 
Table 2 Comparison of the health utility values of gastric cancer patients with socioeconomic characteristics

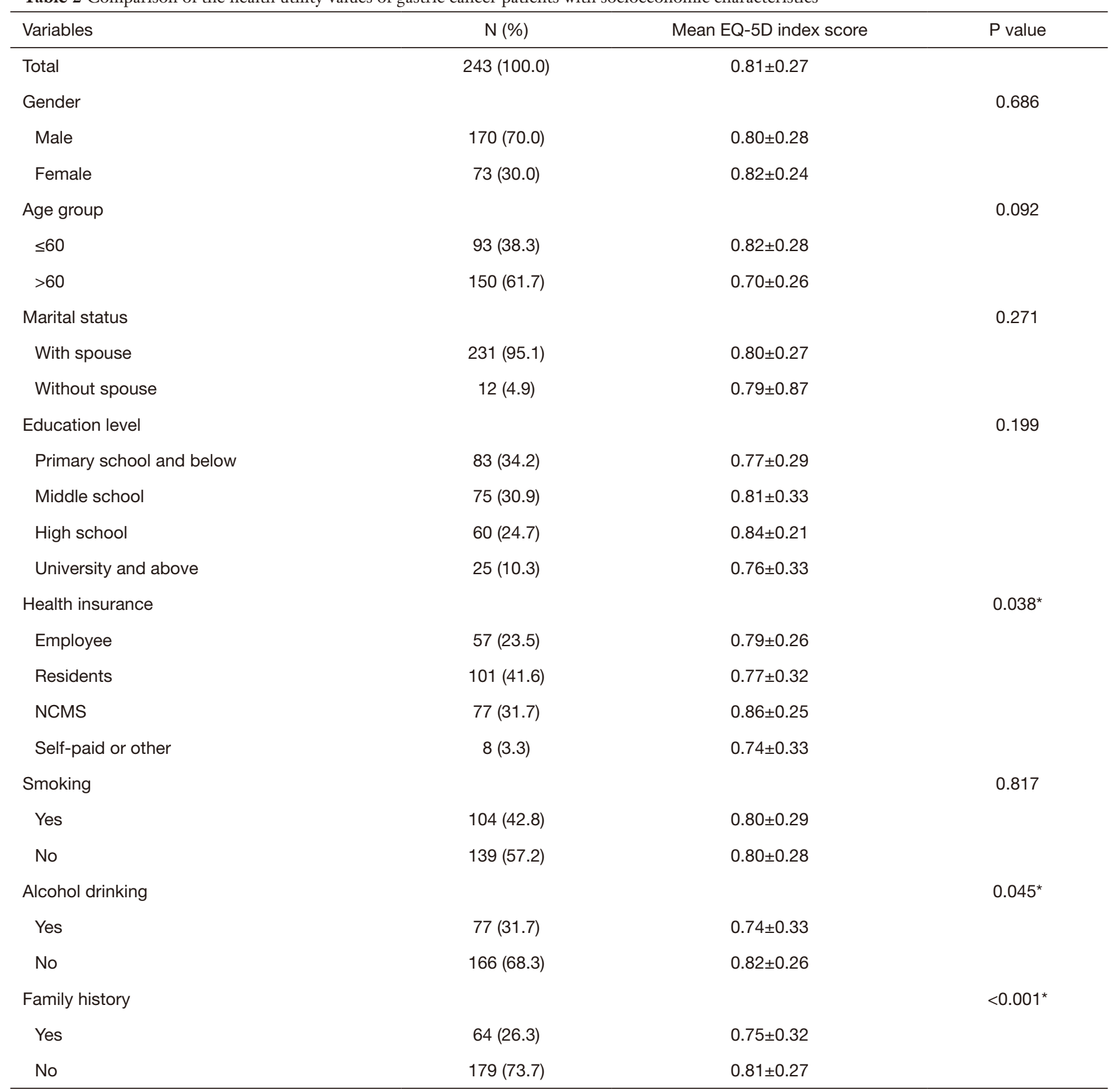

*, $P<0.05$. EQ-5D, EuroQol five-dimension; NCMS, new rural cooperative medical system.

life but also pays more attention to improvements in quality of life (35). In this cross-sectional study, we measured the HRQOL of gastric cancer patients using the Chinese version of the EQ-5D-5L scale and explored the factors that influence patients' quality of life. To the best of our knowledge, only a few studies have assessed the HRQOL of gastric cancer patients in mainland China. HRQOL results can be used to calculate quality-adjusted life years and are essential indicators for health economic evaluations. In addition, we examined five dimensions, including physical and mental problems, which can guide medical staff in targeted intervention measures to improve the quality 
Table 3 Comparison of the health utility values of gastric cancer patients with clinical characteristics

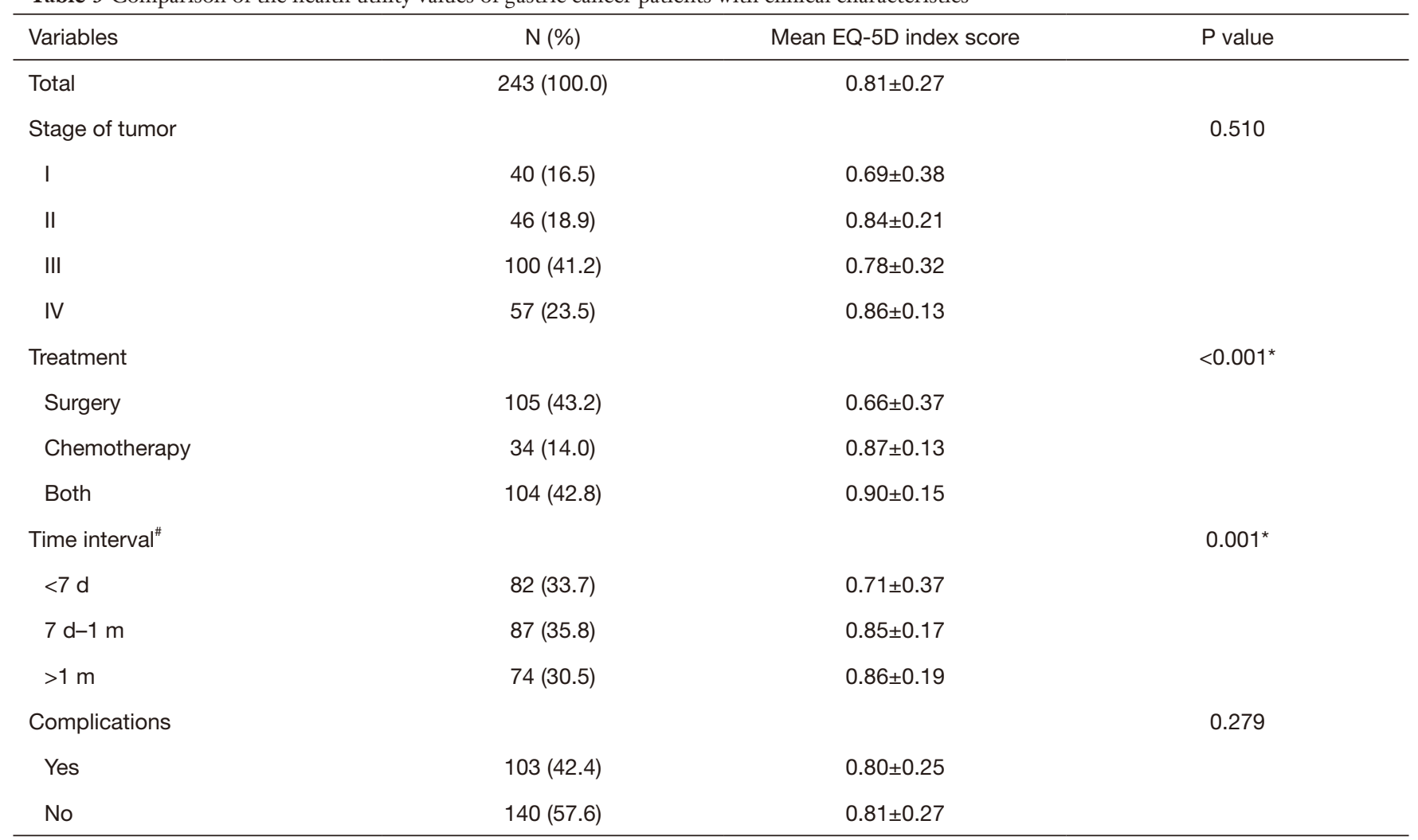

\#, time interval between this survey and last treatment; ${ }^{*}, \mathrm{P}<0.05$. EQ-5D, EuroQol five-dimension.

of life for patients. Furthermore, this study also detected relationships between utility scores and clinical and socioeconomic factors that can help clinicians and policy decision-makers allocate resources more effectively.

In this study, we found that pain/discomfort was the most frequently reported problem among patients $(64.2 \%$, all levels included) in the five health dimensions. The pain/ discomfort experienced may have been due to abdominal discomfort from the cancer itself, the pain following surgery, or the side effects caused by chemotherapy drugs, such as gastrointestinal discomfort, neurovirulence, and hair loss $(36,37)$. Our results highlight the importance of paying more attention to the patient's pain during diagnosis and treatment. If the side effects of chemotherapeutic drugs are severe, other drugs with similar efficacy can be selected as an alternative. Additionally, anxiety/depression was another commonly reported problem (35.4\%, all levels included) in the five dimensions. There is no doubt that psychological problems are significant among cancer patients (38-40). With the extension of treatment time and the increase in treatment costs, the psychological burden for patients becomes greater. Therefore, we should pay more attention to the psychological burden experienced by patients and find strategies to relieve anxiety, improve depressed mood, and help patients build up the confidence to combat the disease and cope with treatment.

Among the socioeconomic factors, we found that whether patients had insurance or not had an impact on their utility scores. The HRQOL of patients who had any health insurance was significantly higher than those who did not. The cost of cancer patients' medical treatment is quite expensive, but with universal medical insurance coverage, patients only need to pay a part of the cost, which greatly reduces their financial burden. As for the factor of medical insurance, clinicians can help patients finish the reimbursement procedures and choose those drugs with larger reimbursement ratio as far as possible, so as to reduce the economic burden of patients. Apart from this, family history was also an important negative factor. It may be that family history interferes with the patient's psychological state, leading them to worry about the health status of other family members. For this factor, we can 
Table 4 Results of the Tobit regression model on EQ-5D-5L index scores of patients

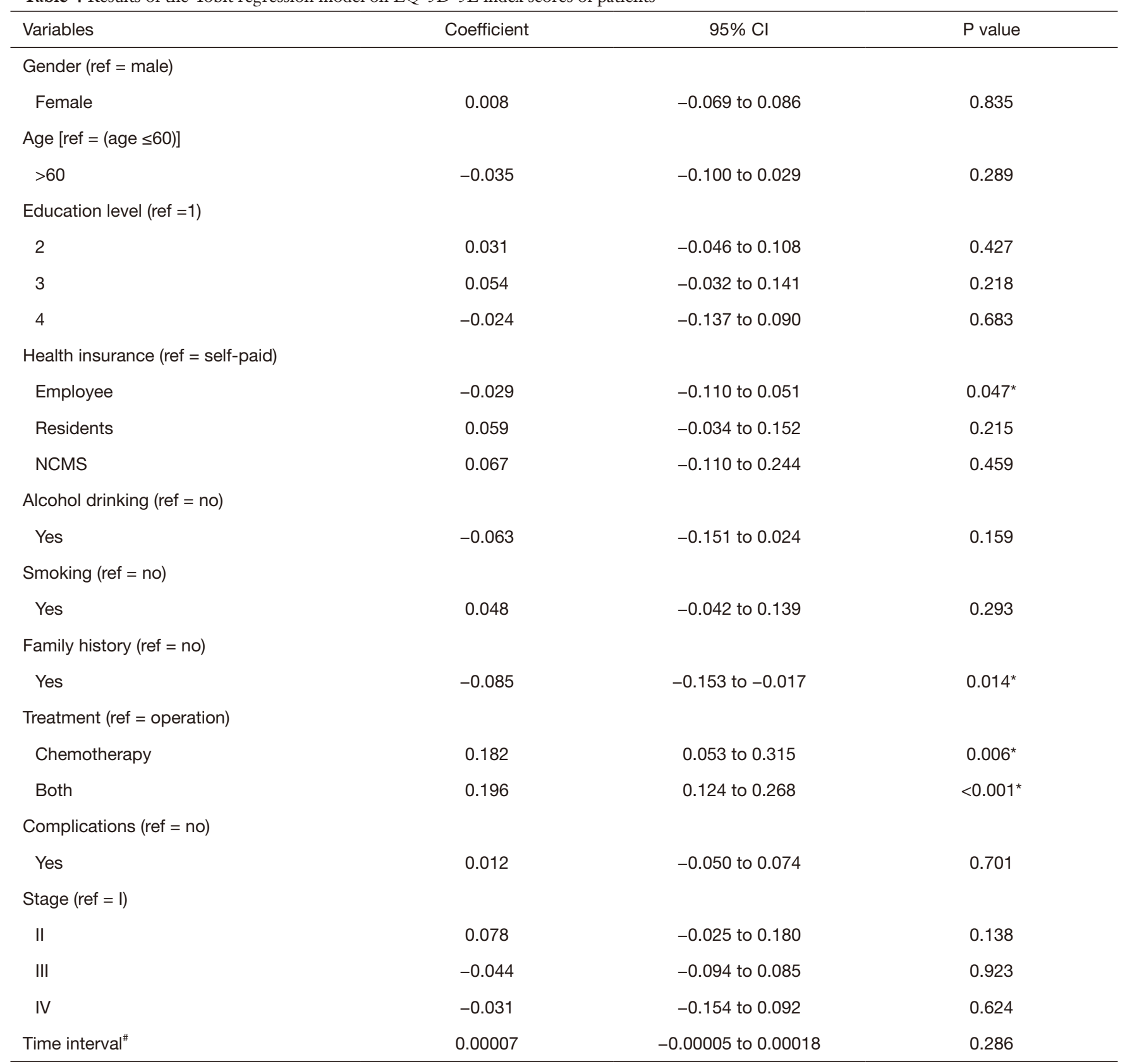

\#, time interval between this survey and last treatment; *, $\mathrm{P}<0.05$. EQ-5D-5L, five-level EuroQol five-dimensional questionnaire; NCMS, new rural cooperative medical system.

calm patients' anxiety, explain the significance of family history, and suggest that the family members have regular medical examinations. However, the Tobit regression model showed that the correlations between gender, age, educational level, and lifestyle with quality of life were not statistically significant. Nevertheless, further investigations of these factors are warranted. In terms of overall scores, our results showed that the HRQOL of female patients was significantly lower than that of male patients. This result is in accord with findings from Yun et al. (41) and Michelson et al. (42). Female patients may be more pessimistic and have a lower tolerance for anxiety, depression, and other negative 
emotions, thus affecting treatment compliance and quality of life. Some studies have also shown that age is related to the quality of life of patients who have gastric cancer, with older patients reporting a worse quality of life $(41,43)$.

In the Tobit regression, the treatment also showed a significant association with the utility scores of gastric cancer patients. Patients who had undergone surgery often had lower HRQOL scores, which may be due to the increased pain and discomfort arising from surgical procedures. On the contrary, patients receiving routine chemotherapy are generally stable with little disruption to their normal lives. Gastric cancer surgery is divided into traditional open surgery and laparoscopic minimally invasive surgery. For patients with gastric cancer, open surgery has great trauma and slow postoperative recovery while laparoscopic minimally invasive surgery has the advantages of minimally invasive, less bleeding and quick recovery (44). Therefore, in the surgical treatment of gastric cancer, doctors should choose laparoscopic surgery as far as possible, which is beneficial to improve the quality of life. There is also some debate about the association between quality of life and the stage of gastric cancer. Some previous studies found that tumor stage had an important influence on the quality of life in patients with gastric cancer and that the HRQOL with stage IV was the poorest (45). But in this study, tumor stage was not significant in the multivariate analysis. We can find that the scores of patients in stage I were the poorest. This may be because patients in this stage were mostly found in general surgery and the interval between the surgery and this research was not long enough to allow for complete wound healing, and their daily activities were significantly affected. However, like many other studies $(46,47)$, most cancer patients in our study were in advanced stages when first diagnosed. Therefore, attention should be paid not only to the tertiary prevention of cancer but also the primary or secondary prevention of gastric cancer. Early screening, early detection, and early treatment of high-risk groups are effective ways to improve the quality of life of gastric cancer patients.

There were several limitations in the study that deserve mention. First, the sample size was moderate because of limited resources, increasing the risk of type II errors. Second, this study was conducted in one hospital in Suzhou, so it was not a representative sample of China. Hence, future studies based on a larger and more representative sample of Chinese gastric cancer patients are warranted. Third, as a cross-sectional survey, our study was unable to establish a causal relationship between gastric cancer and
HRQOL. Last but not least, the information regarding complications and family history was gathered by self-report rather than by medical testing, which may have influenced the accuracy of the results.

\section{Conclusions}

In summary, this research measured the HRQOL of gastric cancer patients by using the EQ-5D-5L and analyzed the influencing factors. Lower scores on the EQ-5D-5L were associated with a lack of health insurance, a family history of cancer, and receiving only surgical treatment. These results may assist in choosing an appropriate individualized treatment plan and strengthening interventions for the main influencing factors, thereby improving patients' quality of life and prolonging their survival period.

\section{Acknowledgments}

We would like to express our gratitude to the study participants for their significant contribution to the data collection.

Funding: This study was supported by General Project of Natural Science Foundation of Higher Education Institutions of Jiangsu Province. Grant/Award Number: 20KJB320027.

\section{Footnote}

Reporting Checklist: The authors have completed the SURGE reporting checklist. Available at https://dx.doi. org/10.21037/apm-21-1599

Data Sharing Statement: Available at https://dx.doi. org/10.21037/apm-21-1599

Conflicts of Interest: All authors have completed the ICMJE uniform disclosure form (available at https://dx.doi. org/10.21037/apm-21-1599). The authors have no conflicts of interest to declare.

Etbical Statement: The authors are accountable for all aspects of the work in ensuring that questions related to the accuracy or integrity of any part of the work are appropriately investigated and resolved. All procedures performed in this study involving human participants were in accordance with the Declaration of Helsinki (as revised in 2013). The study was approved by the Ethics Committee 
of the First Affiliated Hospital of Soochow University of (No. 98, 2021) and informed consent was taken from all the patients.

Open Access Statement: This is an Open Access article distributed in accordance with the Creative Commons Attribution-NonCommercial-NoDerivs 4.0 International License (CC BY-NC-ND 4.0), which permits the noncommercial replication and distribution of the article with the strict proviso that no changes or edits are made and the original work is properly cited (including links to both the formal publication through the relevant DOI and the license). See: https://creativecommons.org/licenses/by-nc-nd/4.0/.

\section{References}

1. Smyth EC, Nilsson M, Grabsch HI, et al. Gastric cancer. Lancet 2020;396:635-48.

2. Chang M, Zhang JC, Zhou Q, et al. Research progress of clinical epidemiology of gastric cancer. Chinese Journal of Gastroenterology and Hepatology 2017;26:966-9.

3. Suzhou Center for Disease Control and Prevention. 2018 Annual Chronic Disease Information Release Report of Suzhou City. Available online: http://wsjkw.suzhou.gov.cn/ szswjw/xwfbh/201912/aa4dcdb3bf3b453a8d932c52247ba 4b4.shtml

4. Paek J, Choi YJ. Association between hand grip strength and impaired health-related quality of life in Korean cancer survivors: a cross-sectional study. BMJ Open 2019;9:e030938.

5. Lee KE, Lim KH. Differences in factors affecting the quality of life over time after the gastrectomy in patients with stage I gastric cancer. Gastroenterol Nurs 2020;43:241-8.

6. Yao X, Hu Y. Current situation of quality of life and related factors in patients with gastric cancer. Chinese Journal of Public Health Engineering 2020;19:525-6.

7. Kundes MF, Kement M, Yegen F, et al. Effects of clinical factors on quality of life following curative gastrectomy for gastric cancer. Niger J Clin Pract 2019;22:661-8.

8. Shi QP, Qin MF, Chen Y, et al. Analysis on quality of life and its influencing factors in Yunnan Province. Chinese Journal of Prevention and Control of Chronic Diseases 2018;26:900-4.

9. Dolan P. Modeling valuations for EuroQol health states. Med Care 1997;35:1095-108.

10. Brazier J, Roberts J, Deverill M. The estimation of a preference-based measure of health from the SF-36. J
Health Econ 2002;21:271-92.

11. Horsman J, Furlong W, Feeny D, et al. The Health Utilities Index (HUI): concepts, measurement properties and applications. Health Qual Life Outcomes 2003;1:54.

12. Brenkman HJF, Tegels JJW, Ruurda JP, et al. Factors influencing health-related quality of life after gastrectomy for cancer. Gastric Cancer 2018;21:524-32.

13. Blazeby JM, Conroy T, Bottomley A, et al. Clinical and psychometric validation of a questionnaire module, the EORTC QLQ-STO 22, to assess quality of life in patients with gastric cancer. Eur J Cancer 2004;40:2260-8.

14. Wang HM, Patrick DL, Edwards TC, et al. Validation of the EQ-5D in a general population sample in urban China. Qual Life Res 2012;21:155-60.

15. Xing YB, Ma AX. Study on reliability and validity of Chinese version of EQ-5D-5L. Shanghai Medical and Pharmaceutical Journal 2013;9:40-3.

16. Wu J, Han Y, Zhao FL, et al. Validation and comparison of EuroQoL-5 dimension (EQ-5D) and Short Form-6 dimension (SF-6D) among stable angina patients. Health Qual Life Outcomes 2014;12:156.

17. Zhao FL, Yue $M$, Yang $H$, et al. Validation and comparison of EuroQol and short form 6D in chronic prostatitis patients. Value Health 2010;13:649-56.

18. Wang P, Luo N, Tai ES, Thumboo J. The EQ-5D-5L is More Discriminative Than the EQ-5D-3L in Patients with Diabetes in Singapore. Value Health Reg Issues 2016;9:57-62.

19. Scalone L, Ciampichini R, Fagiuoli S, et al. Comparing the performance of the standard EQ-5D 3L with the new version EQ-5D 5L in patients with chronic hepatic diseases. Qual Life Res 2013;22:1707-16.

20. Zeng X, Sui M, Liu B, et al. Measurement properties of the EQ-5D-5L and EQ-5D-3L in six commonly diagnosed cancers. Patient 2021;14:209-22.

21. La Mantia I, Rossitto F, Andaloro C. Quality of life in head and neck cancer: Patients' and family caregivers' perceptions. Egyptian Journal of Ear, Nose, Throat and Allied Sciences 2017;18:247-50.

22. Su M, Hua X, Wang J, et al. Health-related quality of life among cancer survivors in rural China. Qual Life Res 2019;28:695-702.

23. Koide R, Kikuchi A, Miyajima M, et al. Quality assessment using EQ-5D-5L after lung surgery for non-small cell lung cancer (NSCLC) patients. Gen Thorac Cardiovasc Surg 2019;67:1056-61.

24. Kameyama H, Shimada Y, Yagi R, et al. Quality of life of patients after colorectal cancer surgery as assessed 
using EQ-5D-5L scores. Gan To Kagaku Ryoho 2017;44:1083-5.

25. Bae JM, Kim S, Kim YW, et al. Health-related quality of life among disease-free stomach cancer survivors in Korea. Qual Life Res 2006;15:1587-96. Erratum in: Qual Life Res 2007;16:913.

26. Lee SS, Chung HY, Kwon OK, et al. Long-term quality of life after distal subtotal and total gastrectomy: symptom- and behavior-oriented consequences. Ann Surg 2016;263:738-44.

27. Xia R, Zeng H, Liu Q, et al. Health-related quality of life and health utility score of patients with gastric cancer: a multi-centre cross-sectional survey in China. Eur J Cancer Care (Engl) 2020;29:e13283.

28. Luo N, Liu G, Li M, et al. Estimating an EQ-5D-5L value set for China. Value Health 2017;20:662-9.

29. Sullivan PW, Ghushchyan VH. EQ-5D scores for diabetesrelated comorbidities. Value Health 2016;19:1002-8.

30. Huang W, Yang J, Liu Y, et al. Assessing health-related quality of life of patients with colorectal cancer using EQ5D-5L: a cross-sectional study in Heilongjiang of China. BMJ Open 2018;8:e022711.

31. Sullivan PW, Ghushchyan VH, Globe G, et al. Health-related quality of life associated with systemic corticosteroids. Qual Life Res 2017;26:1037-58.

32. Pan CW, Sun HP, Zhou HJ, et al. Valuing health-related quality of life in type 2 diabetes patients in China. Med Decis Making 2016;36:234-41.

33. Tran BX, Ohinmaa A, Nguyen LT. Quality of life profile and psychometric properties of the EQ-5D-5L in HIV/ AIDS patients. Health Qual Life Outcomes 2012;10:132.

34. Twisk J, Rijmen F. Longitudinal tobit regression: a new approach to analyze outcome variables with floor or ceiling effects. J Clin Epidemiol 2009;62:953-8.

35. Seo HS, Jung YJ, Kim JH, et al. The effect of metformin on prognosis in patients with locally advanced gastric cancer associated with type 2 diabetes mellitus. Am J Clin Oncol 2019;42:909-17.

36. Zhao XJ, Jiang XL, Peng N, et al. Effect of applying special nursing for emotional status and intestinal side effects of patients with cancer chemotherapy. Nursing

Cite this article as: Zhang FF, Ye L, Yin N, Xiong F, Zhao CH, Zhu YB. Evaluating health-related quality of life in gastric cancer patients in Suzhou, China. Ann Palliat Med 2021;10(7):8024-8033. doi: 10.21037/apm-21-1599
Practice and Research 2020;17:79-81.

37. Xu L. Effects of Two chemotherapy regimens on shortterm efficacy, NLR, PLR and toxic and side effect in patients with metastatic colon carcinoma. Labeled Immunoassays and Clinical Medicine 2018;25:1895-9.

38. Rui XM, Meng XL. Research progress on influencing factors of psychological problems in patients with breast cancer and related intervention measures. Journal of Chinese Oncology 2020;26:1035-40.

39. Grassi L, Spiegel D, Riba M. Advancing psychosocial care in cancer patients. F1000Res 2017;6:2083.

40. Huang GL, Wu YP, Zhang GQ, et, al. Analysis of the psychological conditions and related factors of breast cancer patients. The Chinese-German Journal of Clinical Oncology 2010;9:53-7.

41. Yun YH, Kim SH, Lee KM, et al. Age, sex, and comorbidities were considered in comparing reference data for health-related quality of life in the general and cancer populations. J Clin Epidemiol 2007;60:1164-75.

42. Michelson H, Bolund C, Nilsson B, et al. Healthrelated quality of life measured by the EORTC QLQC30--reference values from a large sample of Swedish population. Acta Oncol 2000;39:477-84.

43. Wang JP, Cui JN, Chen ZG, et al. Quality of life and factors that influence it among cancer patients in China. Chinese Journal of Clinical Psychology 2000;8:23-6.

44. Zhu ZJ, Chen GP. Effect of laparoscopic radical resection on gastrointestinal function and quality of life in patients with gastric cancer. Health Research 2021;41:111-2, 115.

45. Matsushita T, Matsushima E, Maruyama M. Assessment of peri-operative quality of life in patients undergoing surgery for gastrointestinal cancer. Support Care Cancer 2004;12:319-25.

46. Yao Y, Jia YJ, Deng RF, et al. Staging differentiation and treatment for myelosuppression after chemotherapy in gastric cancer patients with traditional Chinese medicine. Journal of Oncology in Chinese Medicine 2020;2:45-9.

47. Ba YI, Zhou LK. The choices of advanced gastric cancer. Chinese Journal of Clinical Oncology 2016;43:21-6.

(English Language Editor: D. Fitzgerald) 